\title{
Violences obstétricales : où en sommes-nous ?
}

\author{
Obstetric Violence: where do We Stand?
}

\begin{abstract}
A. Yamgnane (Obstétricienne, présidente de la commission bientraitance du CNGOF) - C. Donner (Obstétricienne, clinique d'échographie et médecine foetale) · C. Kirkpatrick (Obstétricienne, ancienne responsable du département d'obstétrique)
\end{abstract}

(C) Lavoisier SAS 2020

Ce numéro de la Revue Périnatale a été imaginé comme un point d'étape. Où en sommes-nous au sujet des débats sur le thème « Violences obstétricales »?

Violences obstétricales : le fait de choisir ce titre a suscité au sein de notre comité de rédaction d'intenses débats qui nous ont tenues longtemps en haleine. Nous envisagions de renoncer à utiliser cette expression, car le mot violence souligne l'intentionnalité de nuire, et nous savons que ce choix a rendu le débat inaudible pour nombre des professionnels de santé à qui nous nous adressons. Nous devions parler de ce sujet avec les usagères que nous prétendons protéger, mais comment le faire sans utiliser le même vocable qu'elles ?

Nous avons donc tranché en assumant la part de provocation que certains peut-être ressentiront, et à chaque étape nous avons convoqué Camus : "Mal nommer les choses, c'est ajouter au malheur du monde. Ne pas nommer les choses, c'est nier notre humanité.»"

Nous souhaitions partager avec nos lecteurs ces problématiques complexes sous tous leurs aspects, y compris dans leur point aveugle, mais sans jamais céder à la simplification. Comment ne pas trébucher sur le dilemme sans fin du malentendu inhérent à toute communication. En l'occurrence : il existe un gouffre entre ce qu'éprouve le professionnel dans ses actes, fruit des limites de sa formation, de sa personnalité, de ses conditions d'exercice, et ce que la femme (son partenaire) éprouve en fonction de son parcours et de sa sensibilité propre, et bien sûr de la réalité des gestes et attitudes dans l'environnement médical.

\footnotetext{
A. Yamgnane $(\bowtie)$

Hôpital américain de Paris, 63, boulevard Victor-Hugo, F-92200 Neuilly-sur-Seine, France

e-mail : amina.yamgnane@ahparis.org

C. Donner $(\bowtie) \cdot$ C. Kirkpatrick $(\bowtie)$

Service de gynécologie-obstétrique,

hôpital Erasme, université libre de Bruxelles,

route de Lennik, 808, B-1070 Bruxelles, Belgique

e-mail : cdonner@ulb.ac.be, kichoukirk@gmail.com
}

Autant dire que ce numéro est ambitieux, même s'il ne constitue qu'une première étape dans une réflexion qui doit se poursuivre, car elle traverse tous les champs d'exercice. Nous avons à cœur de féliciter et remercier chacun des auteurs, qui tous ont accepté avec vivacité d'y participer, et qui nous donnent à lire des contenus détaillés et puissants qui ne cèdent jamais à aucun raccourci. Ce numéro permet à chacun de ne plus ignorer que l'émergence de ce débat dans le grand public est en réalité récente dans la déjà très longue histoire que ces violences constituaient aux yeux des professionnels : Baudelocque, inventeur du forceps, s'en préoccupait il y a deux siècles !

Michel Dupuis nous fait prendre de la hauteur en contextualisant les violences obstétricales comme une des violences dans le soin en général sans négliger les problématiques de genre.

Comme en écho, Sylvie Lausberg, historienne, nous interpelle en nous faisant prendre du recul grâce à l'indispensable retour historique sur les problématiques de genre.

Par la voix d'Anne Pardou, pédiatre-réanimateur, les acteurs du champ pédiatrique nous apportent un regard éclairant sur le parcours des tout-petits : les parturientes ne sont pas les seules concernées par la violence au moment de la naissance.

Les études sur les conséquences psychotraumatiques finement étudiées par Antje Horsch, professeur de psychologie, et ses équipes nous démontrent le rôle central que les équipes de périnatalité ont à jouer dans la prévention, la prise en charge et le traitement du syndrome de stress posttraumatique et ses funestes conséquences.

Ghada Hatem, gynécologue et médecin cheffe de la Maison des femmes, partage avec nous son expérience de clinicienne. De sa place de présidente du CEGORIF, elle nous rappelle la nécessaire relativité indispensable au regard à porter lorsque l'on participe à ces réflexions.

Anne Evrard du Ciane nous donne à lire les témoignages des femmes, parfois insoutenables, qui ont tant éprouvé les cliniciens. Le Ciane promeut inlassablement le partage de 
ces lectures avec les professionnels et organise leur lecture dans un climat de sécurité et de confiance. C'est ainsi que le Ciane parvient à se rendre audible, parfois encore difficilement, pour les professionnels et est en mesure de faire émerger des propositions de solutions.

Enfin, Amina Yamgnane, gynécologue-obstétricienne et présidente de la commission de la promotion de la bientraitance au sein du CNGOF, partage avec vous l'expérience de trois ans de travail d'écoute mutuelle et de propositions concrètes accessibles aux professionnels de tous les champs.

Nous avons volontairement donné la parole aux acteurs de terrain, qu'ils soient professionnels ou usagers. Et, ce numéro n'a pas pu faire émerger la question de la place des pères, des coparents ! Cela en dit long aussi sur le chemin qu'il nous reste encore à parcourir. Partie remise : un des numéros à venir sera centré sur les effets de la pandémie à
Covid-19 sur les soins en obstétrique et leur fera la place qui s'impose.

Vos retours seront bienvenus, vos efforts et innovations méritent d'être transmis, car nous sommes là sur le terrain mouvant des subjectivités, à la recherche de nouvelles règles de travail vers un meilleur accordage des uns et des autres. Ce thème d'accordage permet de rappeler, s'il était nécessaire, qu'une expérience traumatique peut entraver durablement la mise en place harmonieuse des rencontres entre parents et bébé. Au-delà des passions soulevées, ou à travers elles, il s'agit bien d'un enjeu majeur : intégrer la dimension émotionnelle dans les pratiques médicales, avec la rigueur nécessaire et les moyens adéquats. L'avenir de l'enfant en dépend.

Bonne lecture. Et partagez ce numéro riche d'enseignement sur les nouvelles pratiques en obstétrique au XxI ${ }^{\mathrm{e}}$ siècle. 